\title{
A MULTICURRENCY EXTENSION OF THE LOGNORMAL INTEREST RATE MARKET MODELS
}

\author{
ERIK SCHLÖGL
}

\begin{abstract}
The Market Models of the term structure of interest rates, in which forward LIBOR or forward swap rates are modelled to be lognormal under the forward probability measure of the corresponding maturity, are extended to a multicurrency setting. If lognormal dynamics are assumed for forward LIBOR or forward swap rates in two currencies, the forward exchange rate linking the two currencies can only be chosen to be lognormal for one maturity, with the dynamics for all other maturities given by no-arbitrage relationships. Alternatively, one could choose forward interest rates in only one currency, say the domestic, to be lognormal and postulate lognormal dynamics for all forward exchange rates, with the dynamics of foreign interest rates determined by no-arbitrage relationships.
\end{abstract}

Since the seminal article of Black and Scholes (1973), their option pricing formula has been applied to a myriad of derivative financial instruments, sometimes on the basis of an arbitrage-free model and sometimes - especially in the hectic world of day-to-day derivatives trading — just on the basis of heuristic analogy. The formula has a strong intuitive appeal for practitioners and remains the most important tool of the financial engineer. Furthermore, market prices for a variety of option contracts are routinely communicated in terms of their Black/Scholes implied volatility, since all other determinants of an option's value, such as the price of the underlying asset and the relevant interest rate, are readily observed elsewhere.

In this context, theoretical work which embeds Black/Scholes-type formulae favored by practitioners in a consistent, arbitrage-free framework is particularly relevant. Miltersen, Sandmann and Sondermann $(1997)^{1}$ (MSS) showed that the practice of pricing interest rate cap and floor contracts by a formula which had hitherto only been justified by analogy to Black/Scholes is consistent with a term structure model satisfying the no-arbitrage constraints of the Heath, Jarrow and Morton (1992) framework. The critical assumption for this result is that relative volatility ${ }^{2}$ of observable market forward rates such as LIBOR, compounded according to market conventions, is deterministic. ${ }^{3}$ Brace, Gatarek and Musiela (1997) (BGM) resolved key questions in the construction of such a model, in particular concerning existence and measure relationships, and coined the term Market Model: It reflects market practice both in interest rate compounding and in the pricing of caps and floors. Given the deterministic volatility assumption, BGM explicitly identified forward LIBOR as lognormal martingales under the forward measure to the end of the respective accrual periods. Thus, they accomplished the paradigm shift toward modelling

Date. This version: May 1, 2001. First version: February 6, 1999. School of Finance and Economics, University of Technology, Sydney, PO Box 123, Broadway, NSW 2007, Australia. E-mail: Erik.Schlogl@uts.edu.au.

The author would like to thank Alan Brace, Marek Musiela, Marek Rutkowski, Daniel Sommer, Catriona March, Darrell Duffie and an anonymous referee for helpful discussions and/or comments, but claims responsibility for any remaining errors.

${ }^{1}$ See also Sandmann and Sondermann (1994) and Sandmann, Sondermann and Miltersen (1995).

${ }^{2}$ The relative volatility of a diffusion process $X(t)$ is $\sigma$ if its quadratic variation is given by $X(t)^{2} \sigma^{2} d t$.

${ }^{3}$ For a succinct treatment of the significance of this assumption, see Rady (1997). 
market observables under the appropriate forward measures, ${ }^{4}$ an approach on which the present paper relies heavily. This paradigm was pursued further by Musiela and Rutkowski (1997a), who also present a particularly straightforward construction of the Market Model. The same methodology can be applied to forward swap rates to derive a model which supports the market practice of pricing swaptions instead of that of pricing caps and floors, as demonstrated by Jamshidian (1997).

The aim of this paper is to extend the Market Models to multiple currencies and to determine to what extent Black/Scholes-type valuation of interest rate caps and floors (or, alternatively, swaptions) can be reconciled with the application of the Black/Scholes formula to options on foreign exchange. We will focus on the core issues of the restrictions that the no-arbitrage requirement imposes on simultaneous lognormality assumptions. These restrictions become particularly clear when the relationships between the martingale measures associated with domestic and foreign numeraire assets are identified. Given a set of lognormality assumptions satisfying these restrictions, obtaining the corresponding closed form option pricing formulae is a straightforward application of the standard techniques of the Black/Scholes framework. ${ }^{5}$

The paper is organized as follows. Section 1 develops the relationships between domestic and foreign equivalent martingale measures and elaborates on the no-arbitrage conditions linking the volatilities of forward exchange and interest rates. The consequences of these conditions for the consistent choice of lognormality assumptions are discussed in section 2. Section 3 covers the continuous tenor case and the implications for the dynamics of the spot exchange rate. Section 4 demonstrates the derivation of closed form and approximate formulae for cross-currency (quanto) instruments in the context of the model. Extensions to Market Model simulation algorithms to accommodate a multicurrency model are presented in section 5 , and section 6 concludes.

\section{Measure Relationships}

Given a filtered probability space $\left(\Omega,\left\{\mathcal{F}_{t}\right\}_{t \in\left[0, T^{*}\right]}, \mathbf{P}_{T^{*}}\right)$ satisfying the usual conditions, let $\left\{W_{T^{*}}(t)\right\}_{t \in\left[0, T^{*}\right]}$ denote a $d$-dimensional standard Wiener process and assume that the filtration $\left\{\mathcal{F}_{t}\right\}_{t \in\left[0, T^{*}\right]}$ is the usual $\mathbf{P}_{T^{*}}$-augmentation of the filtration generated by $\left\{W_{T^{*}}(t)\right\}_{t \in\left[0, T^{*}\right]}$.

The model is set up on the basis of assumptions (BP.1) and (BP.2) of Musiela and Rutkowski (1997a):

(BP.1) For any date $T \in\left[0, T^{*}\right]$, the price process of a zero coupon bond $B(t, T), t \in[0, T]$ is a strictly positive special martingale ${ }^{6}$ under $\mathbf{P}_{T^{*}}$.

(BP.2) For any fixed $T \in\left[0, T^{*}\right]$, the forward process

$$
F_{B}\left(t, T, T^{*}\right)=\frac{B(t, T)}{B\left(t, T^{*}\right)}, \quad \forall t \in[0, T]
$$

follows a martingale under $\mathbf{P}_{T^{*}}$.

\footnotetext{
${ }^{4}$ The forward measure approach to pricing interest rate derivatives dates back to papers by Jamshidian (1987) and Geman (1989).

${ }^{5}$ Independently of the present paper, Mikkelsen (1999) constructs consistent LIBOR Market Model settings under various lognormality assumptions and derives a number of Black/Scholes-type formulae for standard products.

${ }^{6}$ Musiela and Rutkowski (1997a) define a special martingale as a process $X$ which admits a decomposition $X=X_{0}+M+A$, where $X_{0} \in \mathbf{R}, M$ is a real-valued local martingale and $A$ is a real-valued predictable process of finite variation.
} 
The objects to be modelled on the fixed income markets are the $\delta$-compounded forward rates defined by

$$
L(t, T)=\delta^{-1}\left(\frac{B(t, T)}{B(t, T+\delta)}-1\right)
$$

Since the compounding matches the market convention for rates such as the London Interbank Offer Rate, $L(t, T)$ is also referred to as forward LIBOR. Note that by assumption (BP.2), $L\left(t, T^{*}-\delta\right)$ is a martingale under $\mathbf{P}_{T^{*}}$.

Initially, let us consider the discrete-tenor case, i.e. for each of the fixed income markets, the model is constructed in the manner described in section 4.1 of Musiela and Rutkowski (1997a). For notational simplicity, assume a horizon date $T^{*}$ which is a multiple of $\delta$, i.e. $T^{*}=N \delta, N \in \mathbb{N}$, and focus on a finite number of maturities $T_{i}=i \delta, i \in\{0, \ldots, N\}$ (the tenor structure $\mathbb{T}$ ). The dynamics of the (domestic) forward LIBOR rate with the longest maturity under the (domestic) time $T^{*}$ forward probability measure $\mathbf{P}_{T^{*}}$ are given by

$$
d L\left(t, T_{N-1}\right)=L\left(t, T_{N-1}\right) \lambda\left(t, T_{N-1}\right) d W_{T^{*}}(t)
$$

where $W_{T^{*}}$ is a standard Brownian motion with respect to $\mathbf{P}_{T^{*}}$.

Analogously, (BP.1) and (BP.2) are assumed to hold for the foreign fixed income market and under the foreign time $T^{*}$ forward probability measure $\tilde{\mathbf{P}}_{T^{*}}$, we have

$$
d \tilde{L}\left(t, T_{N-1}\right)=\tilde{L}\left(t, T_{N-1}\right) \tilde{\lambda}\left(t, T_{N-1}\right) d \tilde{W}_{T^{*}}(t)
$$

i.e. we use the tilde to denote values on the foreign market. Note that if $\lambda\left(t, T_{N-1}\right)$ is a deterministic function of its arguments, $L(t, T)$ is a lognormal martingale. However, this is not a necessary condition for the model construction.

Consider now the bond price quotients $B(t, T) / B\left(t, T_{i}\right)$ for some $i \in\{1, \ldots, N\}$ and any $T \in\left[0, T^{*}\right]$. Define the time $T_{i}$ forward measure $\mathbf{P}_{T_{i}}$ as the measure under which these bond price quotients are martingales. Since $B(t, T) / B\left(t, T_{i}\right)$ can be interpreted as the price of $B(t, T)$ expressed in terms of units of $B\left(t, T_{i}\right)$, we say that $B\left(t, T_{i}\right)$ is a numeraire and $\mathbf{P}_{T_{i}}$ is the equivalent martingale measure associated with this numeraire. In fact, in order to guarantee the absence of arbitrage in the complete market setting we are considering, the price process of any traded asset expressed in terms of the numeraire must be a martingale under the associated martingale measure. ${ }^{7}$

As shown in Musiela and Rutkowski (1997a), the measures $\mathbf{P}_{T_{i}}$ exist and are linked by the Radon/Nikodym-derivatives given in terms of the Doléans exponential as ${ }^{8}$

$$
\frac{d \mathbf{P}_{T_{i}}}{d \mathbf{P}_{T_{i+1}}}=\mathcal{E}_{T_{i}}\left(\int_{0}^{\cdot} \gamma\left(u, T_{i}, T_{i+1}\right) \cdot d W_{T_{i+1}}(u)\right) \quad \mathbf{P}_{T_{i+1}} \text {-a.s. }
$$

with

$$
\gamma\left(t, T_{i}, T_{i+1}\right)=\frac{\delta L\left(t, T_{i}\right)}{1+\delta L\left(t, T_{i}\right)} \lambda\left(t, T_{i}\right) \quad \forall t \in\left[0, T_{i}\right]
$$

\footnotetext{
${ }^{7}$ This result goes back to a series of papers of Harrison and Kreps (1979), Harrison and Pliska (1981) and Harrison and Pliska (1983), whose seminal work has since then been extended and refined in several ways. For the change of numeraire techniques employed here, the results of Geman, El Karoui and Rochet (1995) are particularly relevant. A more complete list of references can be obtained from any recent book on mathematical finance, such as Musiela and Rutkowski (1997b).
}

${ }^{8}$ The Doléans exponential is given by

$$
\mathcal{E}_{t}\left(\int_{0} \alpha(u) d W(u)\right):=\exp \left\{\int_{0}^{t} \alpha(u) d W(u)-\frac{1}{2} \int_{0}^{t} \alpha^{2}(u) d u\right\}
$$


In particular, we have

$$
d W_{T_{i}}(t)=d W_{T_{i+1}}(t)-\gamma\left(u, T_{i}, T_{i+1}\right) d t
$$

If, for all $i \in\{1, \ldots, N-1\}, \lambda\left(t, T_{i}\right)$ is a deterministic function of its arguments, every process $L\left(t, T_{i}\right)$ is a lognormal martingale under the corresponding probability measure $\mathbf{P}_{T_{i+1}}$ (and analogously for $\tilde{L}\left(t, T_{i}\right)$ under $\left.\tilde{\mathbf{P}}_{T_{i+1}}\right)$.

Now let $X(t)$ denote the spot exchange rate in terms of units of domestic currency per unit of foreign currency. To satisfy technical regularity conditions, let us make the following assumption:

(X.1) The spot exchange rate process $X(t), t \in\left[0, T^{*}\right]$, is a strictly positive special martingale under $\mathbf{P}_{T^{*}}$.

Note that $X(t)$ is not a tradeable asset in either market ${ }^{9}$; thus the spot exchange rate (discounted by the numeraire) will generally not be a martingale under any equivalent martingale measures associated with a numeraire asset. However, the foreign bond converted to domestic currency at the spot exchange rate is a domestic asset, and thus

$$
X\left(t, T_{i}\right):=\frac{\tilde{B}\left(t, T_{i}\right) X(t)}{B\left(t, T_{i}\right)}
$$

is a martingale under $\mathbf{P}_{T_{i}}$. Conversely,

$$
\frac{1}{X\left(t, T_{i}\right)}=\frac{B\left(t, T_{i}\right) \frac{1}{X(t)}}{\tilde{B}\left(t, T_{i}\right)}
$$

is a martingale under $\tilde{\mathbf{P}}_{T_{i}}$. $X\left(t, T_{i}\right)$ is the time $T_{i}$ forward exchange rate.

By the Girsanov transformations (3), all domestic forward measures $\mathbf{P}_{T_{i}}$ are linked to $\mathbf{P}_{T^{*}}$ and all foreign forward measures $\tilde{\mathbf{P}}_{T_{i}}$ are linked to $\tilde{\mathbf{P}}_{T^{*}}$. Since these relationships are transitive, specifying the measure transformation linking $\mathbf{P}_{T_{i}}$ and $\tilde{\mathbf{P}}_{T_{i}}$ fixes the relationships between all forward measures, domestic and foreign.

Without loss of generality (in the sense that we could have chosen any other forward measure instead), let us specify the link for the terminal forward measures. Set

$$
d X\left(t, T^{*}\right)=X\left(t, T^{*}\right) \sigma_{X}\left(t, T^{*}\right) \cdot d W_{T^{*}}(t)
$$

Again, if $\sigma_{X}\left(t, T^{*}\right)$ is a deterministic function of its arguments, $X\left(t, T^{*}\right)$ is a lognormal martingale under $\mathbf{P}_{T^{*}}$.

From general theory we know that in the complete, arbitrage-free market we are considering, there exist unique equivalent measures $\mathbf{P}_{T^{*}}$ and $\tilde{\mathbf{P}}_{T^{*}}$, such that all assets discounted by the time $T^{*}$ domestic zero coupon bond $B\left(\cdot, T^{*}\right)$ are martingales under $\mathbf{P}_{T^{*}}$ and all assets discounted by the foreign bond $\tilde{B}\left(\cdot, T^{*}\right)$ are martingales under $\tilde{\mathbf{P}}_{T^{*}}$. Consequently we must have

$$
\frac{d \tilde{\mathbf{P}}_{T^{*}}}{d \mathbf{P}_{T^{*}}}=\frac{X\left(T^{*}\right) \tilde{B}\left(T^{*}, T^{*}\right) B\left(0, T^{*}\right)}{X(0) \tilde{B}\left(0, T^{*}\right) B\left(T^{*}, T^{*}\right)}=\frac{X\left(T^{*}, T^{*}\right)}{X\left(0, T^{*}\right)}
$$

\footnotetext{
${ }^{9}$ In the context of the model under consideration, a tradeable asset is any semimartingale which can be represented as the value process of a selffinancing trading strategy in domestic and/or foreign zero coupon bonds. For a numeraire asset, we additionally require that the value process is strictly positive. $X(t)$ is only the time $t$ rate of conversion from foreign to domestic currency, i.e. $X(t)$ multiplied by the value of an asset denoted in foreign currency gives the value of that asset denoted in domestic currency.
} 
and restricting $\mathbf{P}_{T^{*}}, \tilde{\mathbf{P}}_{T^{*}}$ to the information given at time $t$ (i.e. for $\mathcal{F}_{t^{-}}$-measurable events), we have

$$
\left.\frac{d \tilde{\mathbf{P}}_{T^{*}}}{d \mathbf{P}_{T^{*}}}\right|_{\mathcal{F}_{t}}=\frac{X\left(t, T^{*}\right)}{X\left(0, T^{*}\right)}
$$

Given the dynamics (7) we chose for the forward foreign/domestic exchange rate $X\left(t, T^{*}\right)$, $\mathbf{P}_{T^{*}}$ and $\tilde{\mathbf{P}}_{T^{*}}$ are therefore linked by

$$
\frac{d \tilde{\mathbf{P}}_{T^{*}}}{d \mathbf{P}_{T^{*}}}=\mathcal{E}_{T^{*}}\left(\int_{0} \sigma_{X}\left(u, T^{*}\right) d W_{T^{*}}(u)\right) \quad \mathbf{P}_{T^{*}-\text { a.s. }}
$$

By Girsanov's Theorem, Brownian motions under the two measures are related by

$$
d \tilde{W}_{T^{*}}(t)=d W_{T^{*}}(t)-\sigma_{X}\left(t, T^{*}\right) d t
$$

and by Ito's lemma,

$$
d\left(\frac{1}{X\left(t, T^{*}\right)}\right)=\frac{1}{X\left(t, T^{*}\right)}\left(-\sigma_{X}\left(t, T^{*}\right) \cdot d W_{T^{*}}(t)+\left\|\sigma_{X}\left(t, T^{*}\right)\right\|^{2} d t\right)
$$

Thus the volatility of the forward domestic/foreign exchange rate $X\left(t, T^{*}\right)^{-1}$ is $\sigma_{\frac{1}{X}}\left(t, T^{*}\right)=$ $-\sigma_{X}\left(t, T^{*}\right)$ and we can write

$$
d\left(\frac{1}{X\left(t, T^{*}\right)}\right)=\frac{1}{X\left(t, T^{*}\right)} \sigma_{\frac{1}{X}}\left(t, T^{*}\right) d \tilde{W}_{T^{*}}(t)
$$

Having linked the foreign and domestic fixed income markets by specifying the volatility of the time $T^{*}$ forward exchange rate under the domestic and foreign time $T^{*}$ forward measures, the volatilities of all other forward exchange rates under the respective forward measures are fixed, since the forward measures of different maturities are already linked by the specification of forward LIBOR volatilities. To derive the remaining forward exchange rate volatilities, we inductively make use of the relationship

$$
\frac{X\left(t, T_{i}\right)}{X\left(t, T_{i+1}\right)}=\frac{B\left(t, T_{i+1}\right)}{B\left(t, T_{i}\right)} \frac{\tilde{B}\left(t, T_{i}\right)}{\tilde{B}\left(t, T_{i+1}\right)}
$$

For ease of notation, consider just the first step of the induction,

$$
X\left(t, T_{N-1}\right)=X\left(t, T^{*}\right) \frac{B\left(t, T^{*}\right)}{B\left(t, T_{N-1}\right)} \frac{\tilde{B}\left(t, T_{N-1}\right)}{\tilde{B}\left(t, T^{*}\right)}
$$

As shown in Musiela and Rutkowski (1997a), the dynamics of the forward bond price are given by

$$
d\left(\frac{B\left(t, T_{N-1}\right)}{B\left(t, T^{*}\right)}\right)=\frac{B\left(t, T_{N-1}\right)}{B\left(t, T^{*}\right)} \gamma\left(t, T_{N-1}, T^{*}\right) \cdot d W_{T^{*}}(t)
$$

By Ito's lemma,

$$
d\left(\frac{B\left(t, T^{*}\right)}{B\left(t, T_{N-1}\right)}\right)=\frac{B\left(t, T^{*}\right)}{B\left(t, T_{N-1}\right)}\left(-\gamma\left(t, T_{N-1}, T^{*}\right) \cdot d W_{T^{*}}(t)+\left\|\gamma\left(t, T_{N-1}, T^{*}\right)\right\|^{2} d t\right)
$$

and for the foreign forward bond, after switching to the corresponding domestic measure

$$
d\left(\frac{\tilde{B}\left(t, T_{N-1}\right)}{\tilde{B}\left(t, T^{*}\right)}\right)=\frac{\tilde{B}\left(t, T_{N-1}\right)}{\tilde{B}\left(t, T^{*}\right)}\left(\tilde{\gamma}\left(t, T_{N-1}, T^{*}\right) \cdot d W_{T^{*}}(t)-\tilde{\gamma}\left(t, T_{N-1}, T^{*}\right) \cdot \sigma_{X}\left(t, T^{*}\right) d t\right)
$$


Thus

$$
\begin{aligned}
d\left(\frac{\tilde{B}\left(t, T_{N-1}\right)}{\tilde{B}\left(t, T^{*}\right)} \frac{B\left(t, T^{*}\right)}{B\left(t, T_{N-1}\right)}\right)= & \frac{\tilde{B}\left(t, T_{N-1}\right)}{\tilde{B}\left(t, T^{*}\right)} \frac{B\left(t, T^{*}\right)}{B\left(t, T_{N-1}\right)}\left(\left(\tilde{\gamma}\left(t, T_{N-1}, T^{*}\right)-\gamma\left(t, T_{N-1}, T^{*}\right)\right) \cdot d W_{T^{*}}(t)\right. \\
& +\left(\left\|\gamma\left(t, T_{N-1}, T^{*}\right)\right\|^{2}-\tilde{\gamma}\left(t, T_{N-1}, T^{*}\right) \cdot \sigma_{X}\left(t, T^{*}\right)\right. \\
& \left.\left.-\gamma\left(t, T_{N-1}, T^{*}\right) \cdot \tilde{\gamma}\left(t, T_{N-1}, T^{*}\right)\right) d t\right)
\end{aligned}
$$

and

$$
\begin{aligned}
d X\left(t, T_{N-1}\right)= & X\left(t, T_{N-1}\right)\left(\left(\tilde{\gamma}\left(t, T_{N-1}, T^{*}\right)-\gamma\left(t, T_{N-1}, T^{*}\right)+\sigma_{X}\left(t, T^{*}\right)\right) \cdot d W_{T^{*}}(t)\right. \\
& \left.-\gamma\left(t, T_{N-1}, T^{*}\right) \cdot\left(\tilde{\gamma}\left(t, T_{N-1}, T^{*}\right)-\gamma\left(t, T_{N-1}, T^{*}\right)+\sigma_{X}\left(t, T^{*}\right)\right) d t\right)
\end{aligned}
$$

Since $d W_{T_{N-1}}=d W_{T^{*}}(t)-\gamma\left(t, T_{N-1}, T^{*}\right) d t$, we have

$$
d X\left(t, T_{N-1}\right)=X\left(t, T_{N-1}\right)\left(\left(\tilde{\gamma}\left(t, T_{N-1}, T^{*}\right)-\gamma\left(t, T_{N-1}, T^{*}\right)+\sigma_{X}\left(t, T^{*}\right)\right) \cdot d W_{T_{N-1}}(t)\right)
$$

Thus we must set

$$
\sigma_{X}\left(t, T_{N-1}\right)=\tilde{\gamma}\left(t, T_{N-1}, T^{*}\right)-\gamma\left(t, T_{N-1}, T^{*}\right)+\sigma_{X}\left(t, T^{*}\right)
$$

i.e. the forward exchange rate volatilities for all maturities are linked by

$$
\sigma_{X}\left(t, T_{i-1}\right)=\tilde{\gamma}\left(t, T_{i-1}, T_{i}\right)-\gamma\left(t, T_{i-1}, T_{i}\right)+\sigma_{X}\left(t, T_{i}\right)
$$

Note that the derivation of (11) does not depend on any of the assumptions of deterministic volatilities, but solely on the fact that $\sigma_{X}, \gamma$ and $\tilde{\gamma}$ are, respectively, the volatility functions of the forward exchange rates, domestic and foreign forward bond prices.

Furthermore, if we choose to link the domestic and foreign market by a forward exchange rate to a maturity other than the terminal, we also solve (11) for $\sigma_{X}\left(t, T_{i}\right)$. In effect, we are using the domestic and foreign forward bond volatilities $\gamma$ and $\tilde{\gamma}$ to move either forward or backward in maturity from the link.

\section{Choices of Lognormality}

As a consequence of (11), the choices of which underlying variables should follow a lognormal probability law are restricted. In the extremes, we have two cases, which can be mixed and matched to produce a variety of "hybrid" models. Consider figures 1 and 2. Each arrow denotes a measure relationship based on a deterministic volatility function, i.e. vertical arrows signify lognormal forward LIBORs while horizontal arrows signify lognormal forward exchange rates.

In figure 1, we have a discrete-tenor lognormal forward LIBOR model for both the domestic and foreign fixed income markets. Furthermore, one forward exchange rate is chosen to be lognormal. With that, all measure relationships are fixed and all remaining forward exchange rate volatilities are given by (11). By inserting (4) into (11), it becomes obvious that these volatilities depend on LIBOR levels and consequently only the forward exchange rate chosen to "link" the two fixed income markets can be lognormal.

More specifically, in this case the model is constructed as follows. We first set up a discrete tenor lognormal forward LIBOR model for each of the currencies as in Musiela and Rutkowski (1997a), by specifying $d$-dimensional volatility vectors $\lambda\left(t, T_{i}\right)$ and $\tilde{\lambda}\left(t, T_{i}\right)$ for all $0 \leq i<N$. Specifying these volatilities completely determines the discrete tenor term structure models in each of the currencies. The measure relationships between forward measures in each currency are constructed by backward induction using equations (3)-(5). Given these relationships, we can move backward and forward in maturity at will. Thus 


\section{Measure Links 1}

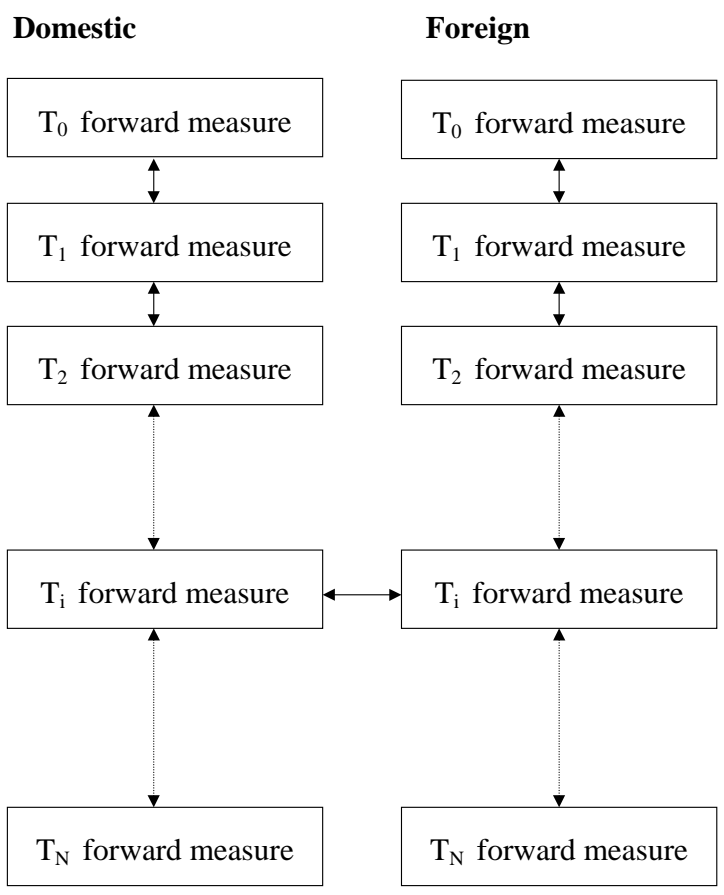

FiguRE 1. Lognormal LIBORs

\section{Measure Links 2}

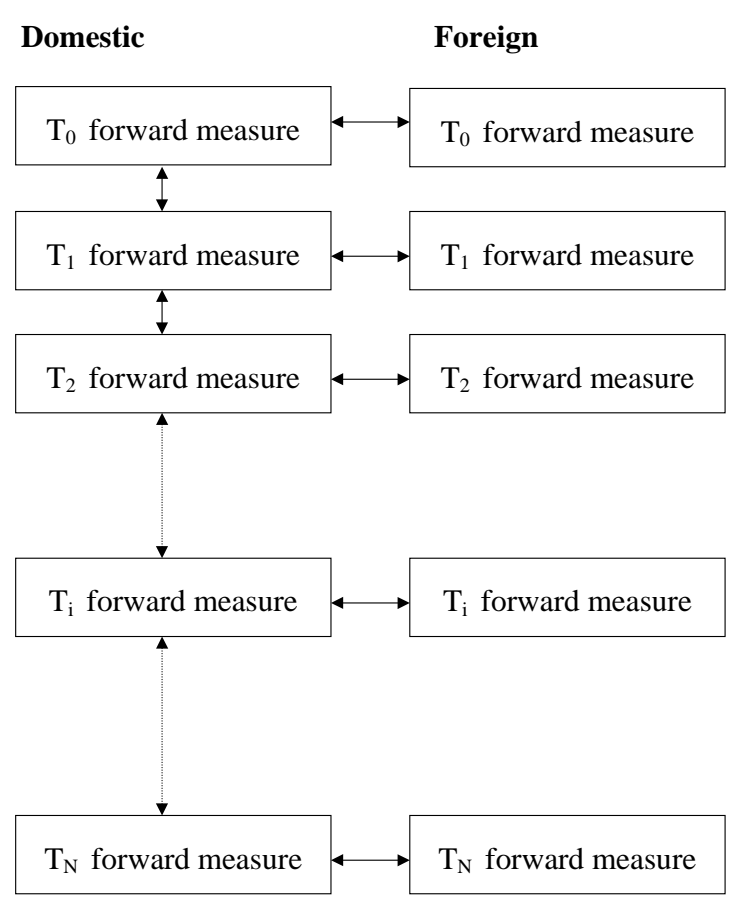

Figure 2. Lognormal exchange rates

it is sufficient to link the two currencies by choosing a forward exchange rate volatility function $\sigma_{X}\left(\cdot, T_{i}\right)$ for some arbitrary $T_{i}$.

In figure 2, only domestic forward LIBORs are assumed to be lognormal, as well as every forward exchange rate. Note that lognormality of the forward exchange rate under the corresponding forward measure means that the Black/Scholes formula holds for a currency option of this maturity, irrespective of the interest rate dynamics. Again, the resulting (discrete-tenor) model is fully specified and condition (11) precludes the lognormality of foreign interest rates.

Here, the domestic discrete tenor model is constructed as in case 1. Furthermore, for all $0 \leq i \leq N$, forward exchange rate volatility functions $\sigma_{X}\left(t, T_{i}\right)$ are input into the model. Given these, (8) permits us to move from any domestic forward measure to the foreign forward measure of the same maturity, and indirectly between foreign forward measures of different maturities, thus all measure relationships are now fixed. By the input volatility function and the measure relationships ${ }^{10}$, the continuous time dynamics of $L\left(t, T_{i}\right)$ and $X\left(t, T_{j}\right)$ are well determined for all $0 \leq i<N$ and $0 \leq j \leq N$, and by equation (9) the $\tilde{L}\left(t, T_{i}\right)$ are well determined also and their dynamics are easily derived by applying Ito's Lemma.

From a practical point of view, it may be attractive to mix the two cases. Consider, for example, a situation where currency options for shorter maturities are very actively traded, while for longer maturities there is very little implied volatility information available in the market. On the other hand, longer-dated interest rate options may be reasonably liquid. In such a case, one could model the "liquid" volatilities as deterministic, greatly facilitating

\footnotetext{
${ }^{10}$ Note that in this context, we could use the terms measure relationship and drift synonymously.
} 
model calibration. This means assuming lognormal forward exchange rates on the short end and moving to lognormal forward interest rates in both markets on the long end, in effect using the volatilities of longer-dated interest rates to extrapolate the volatilities of forward exchange rates.

\section{Continuous Tenor and the Spot Exchange Rate}

The discrete tenor version of the model discussed so far completely specifies stochastic dynamics only for rates (both foreign exchange and interest) maturing at dates in the tenor structure $\mathbb{T}$. Consequently, payoffs that occur at intermediate dates not in $\mathbb{T}$ or depend on intermediate rates cannot be valued. Extending the model to continuous tenor ensures that the value of the numeraire asset is well determined at any point in time and stipulates the dynamics of rates for all intermediate maturities. In particular, this is necessary in order to specify the dynamics of the spot exchange rate $X(t)$.

Case 1: In the continuous tenor version of the Market Models as it was originally proposed in the MSS/BGM papers, the assumption is that all forward LIBORs $L(t, T)$, i.e. for all $t \leq T$ and $T \leq T^{*}-\delta$, are lognormal martingales under the respective forward measures. Then all that is missing are the volatilities of zero coupon bonds with time to maturity less than $\delta$. BGM set this volatility to zero and derive the resulting dynamics of all bond prices $B(t, T)$ and of the continuously compounded short rate $r(t)$.

Case 2: Alternatively, as proposed in Schlögl (1999), one could set the volatility of all bonds maturing strictly before the next date in the tenor structure ${ }^{11}$ to zero and determine these bond prices by a deterministic interpolation scheme. This has the advantage of preserving the Markovian structure of the discrete tenor model and - contrary to the approach taken by MSS/BGM - guaranteeing the non-negativity of all interest rates. Furthermore, it turns out that arbitrage-free interpolation by day-count fractions becomes very tractable.

In either case, if $T_{\eta(t)}$ is the next date in the discrete tenor structure after $t$, the volatility of $B\left(t, T_{\eta(t)}\right)$ is zero. This means that we can speak synonymously of the spot LIBOR measure of Jamshidian (1997) and the risk neutral measure:

3.1. Definition. Given a tenor structure $\mathbb{T}=\left\{T_{i}: i \in\{0,1, \ldots, N\}\right\}$, the spot LIBOR measure $\mathbf{P}_{\rho}$ is the equivalent martingale measure associated with the numeraire

$$
\rho(t)=B\left(t, T_{\eta(t)}\right) \prod_{i=0}^{\eta(t)-1}\left(1+\delta L\left(T_{i}, T_{i}\right)\right)
$$

where

$$
\eta(t)=\min \left\{i \in\{0, \ldots, N\} \mid t \leq T_{i}\right\}
$$

Note that the spot LIBOR measure can be interpreted as a chain of transition probabilities given by conditional forward measures of consecutive maturities (cf. lemma A.1 in the appendix).

3.2. Definition. Given a continuously compounded short rate $r(t)$, the risk neutral measure $\mathbf{P}_{\beta}$ is the equivalent martingale measure associated with the numeraire

$$
\beta(t)=\exp \left\{\int_{0}^{t} r(s) d s\right\}
$$

\footnotetext{
${ }^{11}$ i.e. $B\left(t, T_{\eta(t)}\right)$, with $\eta(t)$ defined as in definition 3.1 below
} 
3.3. LEMMA. If the volatility of $B\left(t, T_{\eta(t)}\right)$ is zero for all $T_{0} \leq t \leq T_{N}$, the spot LIBOR measure and the risk neutral measure coincide when restricted to any $\mathcal{F}_{t}$.

Proof. See appendix. Note that when speaking of the risk neutral measure in this context, it is implicitly assumed that the continuously compounded short rate $r(t)$ exists and is integrable with respect to $t$ almost surely. The key is that the savings account $\beta(t)$ follows a strictly positive, predictable process of finite variation. As argued in Schlögl (1999), if the short bond volatility is zero, the discrete tenor model can always be extended to continuous tenor in such a manner that the short rate exists on each of the open intervals $\left[T_{i}, T_{i+1}[\right.$, which is all that is needed.

If domestic and foreign fixed income markets are described by continuous tenor models of either type 1 or $2, B\left(t, T_{\eta(t)}\right)$ and $\tilde{B}\left(t, T_{\eta(t)}\right)$ are well defined and the dynamics of the spot exchange rate can be obtained (rather tediously) by applying Ito's Lemma to

$$
X(t)=\frac{X\left(t, T_{\eta(t)}\right) B\left(t, T_{\eta(t)}\right)}{\tilde{B}\left(t, T_{\eta(t)}\right)}
$$

$X(t, T)$ can then be calculated for arbitrary $T$ by inserting the $X(t)$ resulting from the above equation into (6).

Note that given BGM's choice setting short bond volatilities to zero, the above relationship implies

$$
\sigma_{X}(t, t)=\sigma_{X}(t, t+\delta)=\sigma_{X}(t, T) \quad \forall t \leq T \leq t+\delta
$$

while in the second case of extending discrete to continuous tenor we only have

$$
\sigma_{X}(t, t)=\sigma_{X}\left(t, T_{\eta(t)}\right)=\sigma_{X}(t, T) \quad \forall t \leq T \leq T_{\eta(t)} \leq t+\delta
$$

Furthermore, since the spot exchange rate $X(t)$ is well defined in a continuous tenor framework, domestic and foreign fixed income markets could be linked by specifying the volatility of $X(t)$. However, stipulating deterministic volatility for the spot exchange rate to link fixed income markets described by continuous tenor MSS/BGM models of cases 1 or 2 is not sufficient to guarantee lognormality of $X(t)$ under the risk neutral measure (i.e. the equivalent martingale measure associated with the continuously compounded savings account), or under any equivalent martingale measure associated with a tradeable numeraire asset, for that matter. This can be seen as a consequence of the highly nonlinear drift term relating forward measures of different maturities (cf. equation 5) and the fact that the spot exchange rate is not a tradeable asset ${ }^{12}$ and therefore not a martingale under any forward measure or the risk neutral measure.

Nor is the lognormality of $X(t)$ a particularly desirable feature with a view to obtaining Black/Scholes-type formulae: For currency options under stochastic interest rates, it is easily verified that a Black/Scholes-type pricing formula results if the exchange rate forward to the maturity of the option is a lognormal martingale under the corresponding forward measure; thus in view of market practice it is more natural to assume deterministic volatilities for forward exchange rates.

If nevertheless one chooses to link fixed income markets in two currencies by a spot exchange rate with deterministic volatility, lognormality under the domestic risk neutral

\footnotetext{
${ }^{12}$ cf. footnote 9
} 
measure holds for the foreign savings account converted to domestic currency at the spot exchange rate: By definition of the risk neutral measure $\mathbf{P}_{\beta}$,

$$
\frac{X(t) \tilde{\beta}(t)}{\beta(t)}=\frac{X(t) \exp \left\{\int_{0}^{t} \tilde{r}(s) d s\right\}}{\exp \left\{\int_{0}^{t} r(s) d s\right\}}
$$

is a martingale under $\mathbf{P}_{\beta}$, where $r$ and $\tilde{r}$ denote the domestic and foreign continuously compounded short rates, respectively. $\tilde{\beta}(t)$ and $\beta(t)$ are of finite variation, thus deterministic volatility of $X(t)$ implies lognormality of $X(t) \tilde{\beta}(t) / \beta(t)$ under $\mathbf{P}_{\beta}$. However, this only implies a Black/Scholes-type formula for the rather exotic option to exchange a foreign savings account for a domestic savings account, i.e. for time $T$ payoffs of the type $[X(T) \tilde{\beta}(T)-K \beta(t)]^{+}$.

As in the discrete tenor version of the model, alternatively to lognormal forward LIBOR dynamics in both currencies, one could specify deterministic volatilities for domestic forward LIBORs only and link fixed income markets in the two currencies by stipulating the volatilities of all exchange rates, forward and spot. The dynamics of foreign zero coupon bonds are then well defined in terms of spot and forward exchange rates, and domestic zero coupon bonds, simply by rearranging (6). Foreign forward LIBOR volatilities are determined by (11), valid in the continuous tenor case for all maturities $t<T \leq T^{*}-\delta$ :

$$
\tilde{\gamma}(t, T, T+\delta)=\sigma_{X}(t, T)-\sigma_{X}(t, T+\delta)+\gamma(t, T, T+\delta)
$$

Furthermore, (8) holds for every maturity, in particular

$$
\begin{aligned}
d \tilde{W}_{\beta}(t) & =d W_{\beta}(t)-\sigma_{X}(t, t) d t \\
d \tilde{W}_{T_{\eta(t)}}(t) & =d W_{T_{\eta(t)}}(t)-\sigma_{X}\left(t, T_{\eta(t)}\right) d t
\end{aligned}
$$

Since the spot LIBOR and risk neutral measures coincide, this implies

$$
d \tilde{W}_{T_{\eta(t)}}(t)=d \tilde{W}_{\beta}(t)+\sigma_{X}(t, t) d t-\sigma_{X}\left(t, T_{\eta(t)}\right) d t
$$

which is not surprising since rearranging (12) yields

$$
\frac{\tilde{B}\left(t, T_{\eta(t)}\right)}{B\left(t, T_{\eta(t)}\right)}=\frac{X\left(t, T_{\eta(t)}\right)}{X(t)}
$$

and applying Ito's lemma to both sides of this equation reveals that the volatility of the foreign short bond $\tilde{B}\left(t, T_{\eta(t)}\right)$ must be $\sigma_{X}\left(t, T_{\eta(t)}\right)-\sigma_{X}(t, t)$. In addition, it is easily verified that the drift of $X(t)$ under $\mathbf{P}_{\beta}$ is $r(t)-\tilde{r}(t)$.

\section{Application Examples}

The most obvious case in which one would resort to a multicurrency term structure model is the pricing of quanto style interest rate derivatives. A large and diverse number of such instruments are becoming increasingly popular. ${ }^{13}$ Two examples serve to illustrate the techniques for derivative pricing in the context of a Multicurrency Lognormal Interest Rate Market Model: the floating-for-floating diff swap and an option on such a swap. While the former can actually be priced in closed form under the appropriate lognormality assumptions, for the latter only an approximate formula can be derived.

\footnotetext{
${ }^{13}$ It is a measure of this popularity that other authors are also actively working on pricing formulae for such products. Independently of the present paper, Pedersen and Miltersen (2000) develop formulae for a range of quanto instruments.
} 
Consider a diff swap to pay 3-month USD LIBOR and receive 3-month EUR LIBOR, with all payments in arrears, in USD, calculated on a USD notional. Let USD be the domestic currency. The value of the diff swap can be expressed as the discounted expectation under the relevant forward measures, i.e.

$$
\begin{aligned}
& \text { notional } \cdot \delta \sum_{i} B\left(0, T_{i+1}\right) E_{T_{i+1}}\left[\tilde{L}\left(T_{i}, T_{i}\right)-L\left(T_{i}, T_{i}\right)\right] \\
= & \operatorname{notional} \cdot \delta \sum_{i} B\left(0, T_{i+1}\right)\left(E_{T_{i+1}}\left[\tilde{L}\left(T_{i}, T_{i}\right)\right]-L\left(0, T_{i}\right)\right)
\end{aligned}
$$

Thus one needs to evaluate $E_{T_{i+1}}\left[\tilde{L}\left(T_{i}, T_{i}\right)\right]$, the expected value of foreign LIBOR under the domestic time $T_{i+1}$ forward measure. The dynamics of $\tilde{L}\left(t, T_{i}\right)$ are given by

$$
\begin{aligned}
d \tilde{L}\left(t, T_{i}\right) & =\tilde{L}\left(t, T_{i}\right) \tilde{\lambda}\left(t, T_{i}\right) d \tilde{W}_{T_{i+1}}(t) \\
& =\tilde{L}\left(t, T_{i}\right) \tilde{\lambda}\left(t, T_{i}\right)\left(d W_{T_{i+1}}(t)-\sigma_{X}\left(t, T_{i+1}\right) d t\right)
\end{aligned}
$$

If one chooses the foreign forward LIBOR volatilities $\tilde{\lambda}$ and the forward exchange rate volatilities $\sigma_{X}$ to be deterministic for all $T_{i}$, the expectation can be evaluated in closed form:

$$
\begin{aligned}
& \tilde{L}\left(t, T_{i}\right)= \tilde{L}\left(0, T_{i}\right) \\
& \cdot \exp \left\{-\int_{0}^{t} \tilde{\lambda}\left(s, T_{i}\right)\left(\sigma_{X}\left(s, T_{i+1}\right)+\frac{1}{2} \tilde{\lambda}\left(s, T_{i}\right)\right) d s\right\} \\
& \cdot \exp \left\{\int_{0}^{t} \tilde{\lambda}\left(s, T_{i}\right) d W_{T_{i+1}}(s)\right\} \\
& \Rightarrow \quad E_{T_{i+1}}\left[\tilde{L}\left(T_{i}, T_{i}\right)\right]=\tilde{L}\left(0, T_{i}\right) \exp \left\{-\int_{0}^{T_{i}} \tilde{\lambda}\left(s, T_{i}\right) \sigma_{X}\left(s, T_{i+1}\right) d s\right\}
\end{aligned}
$$

Thus the value of the diff swap is ${ }^{14}$

$$
\text { notional } \cdot \delta \cdot \sum_{i} B\left(0, T_{i+1}\right)\left(\tilde{L}\left(0, T_{i}\right) \exp \left\{-\int_{0}^{T_{i}} \tilde{\lambda}\left(s, T_{i}\right) \sigma_{X}\left(s, T_{i+1}\right) d s\right\}-L\left(0, T_{i}\right)\right)
$$

Alternatively, one may for some reason ${ }^{15}$ have chosen the domestic forward LIBOR volatilities $\lambda$ to be deterministic. In this case the problem of evaluating $E_{T_{i+1}}\left[\tilde{L}\left(T_{i}, T_{i}\right)\right]$ no longer lends itself to a closed form solution. Writing the dynamics of $\tilde{L}\left(t, T_{i}\right)$ in terms of the deterministic volatility functions $\lambda$ and $\sigma_{X}$ yields

$$
\frac{d \tilde{L}\left(t, T_{i}\right)}{\tilde{L}\left(t, T_{i}\right)}=\frac{1+\delta \tilde{L}\left(t, T_{i}\right)}{\delta \tilde{L}\left(t, T_{i}\right)}\left(\sigma_{X}\left(t, T_{i}\right)-\sigma_{X}\left(t, T_{i+1}\right)+\frac{\delta L\left(t, T_{i}\right)}{1+\delta L\left(t, T_{i}\right)} \lambda\left(t, T_{i}\right)\right) d \tilde{W}_{T_{i+1}}(t)
$$

In order to obtain an approximate solution, one can employ the argument which was first used to derive an approximate swaption formula in a lognormal forward LIBOR model. ${ }^{16}$

\footnotetext{
${ }^{14}$ Note that in general, the driving Brownian motions are multidimensional and the volatilities $\lambda\left(t, T_{i}\right)$, $\tilde{\lambda}\left(t, T_{i}\right)$ and $\sigma_{X}\left(t, T_{i}\right)$ are vectors. The correlation between interest rates and exchange rates thus enters the pricing equation via the scalar products of volatility vectors. For a more explicit discussion of these correlations, see Pedersen and Miltersen (2000).

${ }^{15}$ Ease of calibration is one potential reason which springs to mind, e.g. in a situation where domestic caps and floors are the most liquid source of interest rate volatility information.

${ }^{16}$ This argument first appears in Brace, Gatarek and Musiela (1997). It was developed further in Brace, Dun and Barton (1998a) and formalized by Brace and Womersley (2000).
} 
Noting that the quotient $\delta L\left(t, T_{i}\right) /\left(1+\delta L\left(t, T_{i}\right)\right)$ has low variance under the time $T_{i+1}$ forward measure, it is substituted by its initial value $\delta L\left(0, T_{i}\right) /\left(1+\delta L\left(0, T_{i}\right)\right)$ to yield the SDE

$$
\begin{aligned}
d \tilde{L}\left(t, T_{i}\right) \approx & \left(\frac{1}{\delta}+\tilde{L}\left(t, T_{i}\right)\right) \\
& \underbrace{\left(\sigma_{X}\left(t, T_{i}\right)-\sigma_{X}\left(t, T_{i+1}\right)+\frac{\delta L\left(0, T_{i}\right)}{1+\delta L\left(0, T_{i}\right)} \lambda\left(t, T_{i}\right)\right)}_{\tilde{\lambda}_{\operatorname{approx}}\left(t, T_{i}\right)} d \tilde{W}_{T_{i+1}}(t)
\end{aligned}
$$

which is solved explicitly by

$$
\begin{aligned}
\tilde{L}\left(t, T_{i}\right)= & \left(\frac{1}{\delta}+\tilde{L}\left(0, T_{i}\right)\right) \exp \left\{\int_{0}^{t} \tilde{\lambda}_{\text {approx }}\left(s, T_{i}\right) d W_{T_{i+1}}(s)\right. \\
& \left.-\int_{0}^{t} \tilde{\lambda}_{\text {approx }}\left(s, T_{i}\right)\left(\sigma_{X}\left(s, T_{i+1}\right)+\frac{1}{2} \tilde{\lambda}_{\text {approx }}\left(s, T_{i}\right)\right) d s\right\}-\frac{1}{\delta}
\end{aligned}
$$

Then,

$$
E_{T_{i+1}}\left[\tilde{L}\left(t, T_{i}\right)\right] \approx\left(\frac{1}{\delta}+\tilde{L}\left(0, T_{i}\right)\right) \exp \left\{-\int_{0}^{t} \tilde{\lambda}_{\text {approx }}\left(s, T_{i}\right) \sigma_{X}\left(s, T_{i+1}\right) d s\right\}-\frac{1}{\delta}
$$

Using the same type of approximation argument, the diff swap can also be priced under the assumption that both domestic and foreign forward LIBOR volatilities are deterministic.

Consider now an option on a floating-for-floating diff swap, with the option expiring at time $T_{0}$. This can be generically valued as

$$
B\left(0, T_{0}\right) E_{T_{0}}\left[\max \left(0, \text { notional } \cdot \delta \cdot \sum_{i=1}^{n} B\left(T_{0}, T_{i}\right)\left(E_{T_{i}}\left[\tilde{L}\left(T_{i-1}, T_{i-1}\right) \mid \mathcal{F}_{T_{0}}\right]-L\left(T_{0}, T_{i-1}\right)\right)\right)\right]
$$

This can no longer be calculated in closed form for any permissible combination of choices for deterministic volatilities from $\lambda, \tilde{\lambda}$ and $\sigma_{X}$. To illustrate the technique for deriving an approximate pricing formula, choose $\lambda$ and $\sigma_{X}$ to be deterministic. Then (15) becomes

$$
\begin{aligned}
B\left(0, T_{0}\right) E_{T_{0}} & {\left[\operatorname { m a x } \left(0 , \text { notional } \cdot \delta \cdot \sum _ { i = 1 } ^ { n } B ( T _ { 0 } , T _ { i } ) \left(\left(\frac{1}{\delta}+\tilde{L}\left(T_{0}, T_{i-1}\right)\right)\right.\right.\right.} \\
& \left.\left.\left.\cdot \exp \left\{\int_{T_{0}}^{T_{i-1}} \tilde{\lambda}_{\text {approx }}\left(s, T_{i-1}\right) \sigma_{X}\left(s, T_{i}\right) d s\right\}-\frac{1}{\delta}-L\left(T_{0}, T_{i-1}\right)\right)\right)\right]
\end{aligned}
$$

Define

$$
\begin{aligned}
\bar{\omega}(t) & :=\frac{\sum_{i=1}^{n} B\left(t, T_{i}\right)\left(\left(\frac{1}{\delta}+\tilde{L}\left(t, T_{i-1}\right)\right) \exp \left\{-\int_{t}^{T_{i-1}} \tilde{\lambda}_{\text {approx }}\left(s, T_{i-1}\right) \sigma_{X}\left(s, T_{i}\right) d s\right\}-\frac{1}{\delta}\right)}{\sum_{i=1}^{n} B\left(t, T_{i}\right)} \\
\omega(t) & :=\frac{\sum_{i=1}^{n} B\left(t, T_{i}\right) L\left(t, T_{i-1}\right)}{\sum_{i=1}^{n} B\left(t, T_{i}\right)}
\end{aligned}
$$


Note that the latter is a domestic forward swap rate. Now (15) can be written as

$$
\begin{aligned}
& \text { notional } \cdot \delta \cdot B\left(0, T_{0}\right) E_{T_{0}}\left[\sum_{i=1}^{n} B\left(T_{0}, T_{i}\right)\left[\bar{\omega}\left(T_{0}\right)-\omega\left(T_{0}\right)\right]^{+}\right] \\
= & \operatorname{notional} \cdot \delta \cdot\left(\sum_{i=1}^{n} B\left(0, T_{i}\right)\right) E_{\omega}\left[\left[\bar{\omega}\left(T_{0}\right)-\omega\left(T_{0}\right)\right]^{+}\right]
\end{aligned}
$$

where $E_{\omega}$ is the expectation operator under the forward swap measure $\mathbf{P}_{\omega}$, i.e. the martingale measure associated with the numeraire $\sum_{i=1}^{n} B\left(\cdot, T_{i}\right)$. Clearly, $\omega(t)$ is a martingale under this measure: ${ }^{17}$

$$
\frac{d \omega(t)}{\omega(t)}=\sigma_{\omega}(t) d W_{\omega}(t)
$$

Brace and Womersley (2000) derive the forward swap rate volatility as

$$
\sigma_{\omega}(t)=\sum_{j=1}^{n} A_{j}(t) \lambda\left(t, T_{j-1}\right)
$$

where

$$
A_{j}(t)=\frac{B\left(t, T_{j}\right) L\left(t, T_{j-1}\right)}{\sum_{i=1}^{n} B\left(t, T_{i}\right) L\left(t, T_{i-1}\right)}+\frac{\delta L\left(t, T_{j-1}\right)}{1+\delta L\left(t, T_{j-1}\right)} \sum_{\ell=j}^{n}\left[\frac{B\left(t, T_{\ell}\right)}{\sum_{i=1}^{n} B\left(t, T_{i}\right)}-\frac{B\left(t, T_{\ell}\right) L\left(t, T_{\ell-1}\right)}{\sum_{i=1}^{n} B\left(t, T_{i}\right) L\left(t, T_{i-1}\right)}\right]
$$

By noting that the components of $A_{j}(t)$ are low variance martingales, Brace/Womersley justify replacing $A_{j}(t)$ by its initial value $A_{j}(0)$, i.e.

$$
\sigma_{\omega}(t)=\sum_{j=1}^{n} A_{j}(0) \lambda\left(t, T_{j-1}\right)
$$

resulting in a lognormal martingale for $\omega(t)$ under $\mathbf{P}_{\omega}$. This is consistent with the finding of Brace, Dun and Barton (1998a), that forward swap rates are statistically lognormal in the lognormal forward LIBOR model.

Consider now $\bar{\omega}(t)$. By the solution (14) to the dynamics of foreign forward LIBOR,

$$
\begin{gathered}
\left(\frac{1}{\delta}+\tilde{L}\left(t, T_{i-1}\right)\right) \exp \left\{\int_{0}^{t} \tilde{\lambda}_{\text {approx }}\left(s, T_{i-1}\right) \sigma_{X}\left(s, T_{i}\right) d s\right\} \\
(17)=\left(\frac{1}{\delta}+\tilde{L}\left(0, T_{i-1}\right)\right) \exp \left\{\int_{0}^{t} \tilde{\lambda}_{\text {approx }}\left(s, T_{i-1}\right) d W_{T_{i}}(s)-\frac{1}{2} \int_{0}^{t} \tilde{\lambda}_{\text {approx }}^{2}\left(s, T_{i-1}\right) d s\right\}
\end{gathered}
$$

where the terms on either side of (17) are $\mathbf{P}_{T_{i}}-$ martingales. Now,

$$
\begin{aligned}
&\left(\frac{1}{\delta}+\tilde{L}\left(t, T_{i-1}\right)\right) \exp \left\{-\int_{t}^{T_{i-1}} \tilde{\lambda}_{\text {approx }}\left(s, T_{i-1}\right) \sigma_{X}\left(s, T_{i}\right) d s\right\} \\
&=\left(\frac{1}{\delta}+\tilde{L}\left(t, T_{i-1}\right)\right) \exp \left\{\int_{0}^{t} \tilde{\lambda}_{\text {approx }}\left(s, T_{i-1}\right) \sigma_{X}\left(s, T_{i}\right) d s\right\} \\
& \cdot \exp \left\{-\int_{0}^{T_{i-1}} \tilde{\lambda}_{\text {approx }}\left(s, T_{i-1}\right) \sigma_{X}\left(s, T_{i}\right) d s\right\}
\end{aligned}
$$

where both sides of (18) are still $\mathbf{P}_{T_{i}}$-martingales, since we have simply multiplied a martingale by a deterministic factor independent of $t$. By multiplying each term in the sum in the numerator of $\bar{\omega}(t)$ by the corresponding numeraire $B\left(t, T_{i}\right)$ and dividing the result by

\footnotetext{
${ }^{17}$ The forward swap measure was first studied by Jamshidian (1997).
} 
the denominator, one has in effect changed numeraires to $\sum_{i=1}^{n} B\left(t, T_{i}\right)$. Therefore, $\bar{\omega}(t)$ is also a $\mathbf{P}_{\omega}$-martingale. To shorten notation, set

$$
\begin{aligned}
B_{i} & :=B\left(t, T_{i}\right) \\
\bar{B}_{i} & :=B\left(t, T_{i}\right) \exp \left\{-\int_{t}^{T_{i-1}} \tilde{\lambda}_{\text {approx }}\left(s, T_{i-1}\right) \sigma_{X}\left(s, T_{i}\right) d s\right\} \\
\tilde{L}_{i} & :=\tilde{L}\left(t, T_{i}\right) \\
\tilde{\lambda}_{i} & :=\tilde{\lambda}_{\text {approx }}\left(t, T_{i}\right) \\
\sigma_{F}^{(i)} & :=\sum_{\ell=1}^{i} \frac{\delta L\left(t, T_{\ell-1}\right)}{1+\delta L\left(t, T_{\ell-1}\right)} \lambda\left(t, T_{\ell-1}\right)
\end{aligned}
$$

Applying Ito's lemma, the volatility of $\bar{\omega}(t)$ can be written as

$$
\frac{\sum_{i=1}^{n}\left(\bar{B}_{i}\left(\frac{1}{\delta}+\tilde{L}_{i-1}\right) \tilde{\lambda}_{i-1}+B_{i} \sigma_{F}^{(i)} \frac{\sum_{j=1}^{n} \bar{B}_{j}\left(\frac{1}{\delta}+\tilde{L}_{j-1}\right)}{\sum_{j=1}^{n} B_{j}}-\left(\frac{1}{\delta}+\tilde{L}_{i-1}\right) \bar{B}_{i} \sigma_{F}^{(i)}\right)}{\sum_{i=1}^{n}\left(\bar{B}_{i}\left(\frac{1}{\delta}+\tilde{L}_{i-1}\right)-\frac{1}{\delta} B_{i}\right)}
$$

To justify substituting the $B\left(t, T_{i}\right), L\left(t, T_{i}\right)$ and $\tilde{L}\left(t, T_{i}\right)$ in the above expression by their time 0 values, consider the following:

$$
\begin{aligned}
\sum_{i=1}^{n} B_{i} \sigma_{F}^{(i)} \frac{\sum_{j=1}^{n} \bar{B}_{j}\left(\frac{1}{\delta}+\tilde{L}_{j-1}\right)}{\sum_{j=1}^{n} B_{j}} & \approx \sum_{i=1}^{n}\left(\frac{1}{\delta}+L_{i-1}\right) \bar{B}_{i} \sigma_{F}^{(i)} \\
\Leftrightarrow\left(\sum_{i=1}^{n} B_{i} \sigma_{F}^{(i)}\right) \sum_{j=1}^{n} \bar{B}_{j}\left(\frac{1}{\delta}+\tilde{L}_{j-1}\right) & \approx\left(\sum_{j=1}^{n} B_{j}\right) \sum_{i=1}^{n}\left(\frac{1}{\delta}+L_{i-1}\right) \bar{B}_{i} \sigma_{F}^{(i)}
\end{aligned}
$$

On either side of (19) we have slightly differently weighted sums of the forward bond price volatilities $\sigma_{F}^{(i)}$. Any changes in these terms due to changes in $B\left(t, T_{i}\right), L\left(t, T_{i}\right)$ and $\tilde{L}\left(t, T_{i}\right)$ will tend to cancel. Similarly, any such changes in the numerator $\sum_{i=1}^{n} \bar{B}_{i}\left(\delta^{-1}+\tilde{L}_{i-1}\right) \tilde{\lambda}_{i-1}$ will be mostly compensated by corresponding changes in the denominator $\sum_{i=1}^{n}\left(\bar{B}_{i}\left(\delta^{-1}+\right.\right.$ $\left.\left.\tilde{L}_{i-1}\right)-\delta^{-1} B_{i}\right)$.

Given approximate deterministic volatilities for $\omega(t)$ and $\bar{\omega}(t)$, the option on a diff swap can be priced by the Black/Scholes-type formula

notional •

$$
\begin{aligned}
& \left(\left(\sum_{i=1}^{n} B\left(0, T_{i}\right)\left(\left(1+\delta \tilde{L}\left(0, T_{i-1}\right)\right) \exp \left\{-\int_{0}^{T_{i-1}} \tilde{\lambda}_{\text {approx }}\left(s, T_{i-1}\right) \sigma_{X}\left(s, T_{i}\right) d s\right\}-1\right)\right) \cdot \mathcal{N}\left(h_{1}\right)\right. \\
& \left.\quad-\left(B\left(0, T_{0}\right)-B\left(0, T_{n}\right)\right) \mathcal{N}\left(h_{2}\right)\right)
\end{aligned}
$$

with

$$
h_{1,2}=\frac{\ln \frac{\bar{\omega}(0)}{\omega(0)} \pm \frac{1}{2} \int_{0}^{T_{0}}\left(\sigma_{\bar{\omega}}(s)-\sigma_{\omega}(s)\right)^{2} d s}{\sqrt{\int_{0}^{T_{0}}\left(\sigma_{\bar{\omega}}(s)-\sigma_{\omega}(s)\right)^{2} d s}}
$$


where $\sigma_{\omega}(\cdot)$ is defined in $(16)$ and

$$
\begin{aligned}
\sigma_{\bar{\omega}}(t) & :=\sum_{i=1}^{n}\left(\bar{A}_{i}(0) \lambda\left(t, T_{i-1}\right)+\tilde{A}_{i}(0) \tilde{\lambda}_{\text {approx }}\left(t, T_{i-1}\right)\right) \\
\bar{A}_{i}(t) & :=\frac{\frac{\delta L_{i-1}}{1+\delta L_{i-1}} \sum_{\ell=i}^{n}\left(\frac{\sum_{j=1}^{n} \bar{B}_{j}\left(\frac{1}{\delta}+\tilde{L}_{j-1}\right)}{\sum_{j=1}^{n} B_{j}} B_{\ell}-\left(\frac{1}{\delta}+\tilde{L}_{\ell-1}\right) \bar{B}_{\ell}\right)}{\sum_{j=1}^{n}\left(\bar{B}_{j}\left(\frac{1}{\delta}+\tilde{L}_{j-1}\right)-\frac{1}{\delta} B_{j}\right)} \\
\tilde{A}_{i}(t) & :=\frac{\bar{B}_{i}\left(\frac{1}{\delta}+\tilde{L}_{i-1}\right)}{\sum_{j=1}^{n}\left(\bar{B}_{j}\left(\frac{1}{\delta}+\tilde{L}_{j-1}\right)-\frac{1}{\delta} B_{j}\right)}
\end{aligned}
$$

Thus one has obtained an approximate pricing formula for an option on a floating-forfloating diff swap in a model where domestic forward LIBOR volatilities $\lambda$ and forward exchange rate volatilities $\sigma_{X}$ are deterministic. Using the same approach, this option could be priced under other lognormality assumptions. ${ }^{18}$ Also, instead of a floating-forfloating diff swap, one could consider diff swaps (and the corresponding options) where one or both parties pay a fixed rate. Applying the above techniques to such contracts is straightforward, and actually simpler than in the floating-for-floating case, as the value of the fixed side of a diff swap is trivial to determine.

The lognormal approximations used to develop the diff swaption formula mirror those made in the derivation of the swaption formula in the single currency case, where they have been shown to be suitable for both pricing and hedging ${ }^{19}$. In the multicurrency case, this approach also appears reasonable and serves to illustrate how one might apply the volatility and measure relationships derived here. While beyond the scope of the present paper and thus left to further research, a comprehensive simulation study would be useful to verify the accuracy of the approximations in a multicurrency setting.

\section{Simulation Algorithms}

As discussed above, consistency with no-arbitrage conditions restricts the lognormality assumptions which can be made simultaneously, leaving some standard products and of course the more complex derivative instruments to be priced numerically or by approximate formulae. The non-linear drift terms relating Brownian motions under forward measures of different maturities preclude any simple Markovian structure of the model ${ }^{20}$ and thus Monte Carlo simulation becomes the numerical method of choice in many cases. This section serves to briefly outline how two simulation algorithms proposed in the literature can be easily extended to accommodate the multicurrency case.

Glasserman and Zhao (2000) present an algorithm in which forward LIBORs (or forward swap rates) are first transformed into variables which are martingales under the forward measure chosen for simulation. These variables are then simulated and forward LIBORs are subsequently recovered from the simulated values. By simulating martingales, Glasserman

\footnotetext{
${ }^{18}$ Clearly one could also take an approach along the lines of Jamshidian (1997) to construct a model where an option on a floating-for-floating diff swap can be priced exactly by a Black/Scholes-type formula. However, the calculations of measure changes and volatilities in such a model would become extremely tedious and it does not seem worthwhile to consider such a highly specialized model.

${ }^{19}$ cf. Brace, Dun and Barton (1998a) and Dun, Schlögl and Barton (2001)

${ }^{20}$ For a discussion of the Markovian properties of the lognormal Market Models, see Schlögl (1999).
} 
and Zhao avoid the bias that results from the discretization of a stochastic drift term. To apply their approach, suitably transformed variables need to be specified.

First, let us consider a discrete tenor model ${ }^{21}$ with both domestic and foreign lognormal forward LIBORs. Assume the simulation is to be carried out under the domestic time $T_{n}$ forward measure (the case for a simulation under a foreign measure is covered by a symmetric argument). As in Glasserman/Zhao, the variable

$$
V_{n}\left(t, T_{j}\right)= \begin{cases}L\left(t, T_{j}\right) \prod_{i=j+1}^{n-1}\left(1+\delta L\left(t, T_{i}\right)\right) & j=1, \ldots, n-1 \\ L\left(t, T_{j}\right) \prod_{i=n \vee \eta(t)}^{j}\left(1+\delta L\left(t, T_{i}\right)\right)^{-1} & j=n, \ldots, N-1\end{cases}
$$

is a martingale under the $T_{n}$ forward measure up to $T_{n}$, and under the spot LIBOR measure thereafter. Given $V_{n}\left(t, T_{j}\right)$ for all $j \in\{1, \ldots, N-1\}$, all domestic forward LIBORs $L\left(t, T_{j}\right)$ can be recovered.

Analogously defined, $\tilde{V}_{n}\left(t, T_{j}\right)$ is a martingale under the foreign time $T_{n}$ forward measure up to $T_{n}$, and under the foreign spot LIBOR measure thereafter. Consequently, $X\left(t, T_{n \vee \eta(t)}\right) \tilde{V}_{n}\left(t, T_{j}\right)$ is a martingale under the domestic measure, as is $X\left(t, T_{n \vee \eta(t)}\right)$. Therefore, $X\left(t, T_{n \vee \eta(t)}\right)$ is simulated directly, and given $X\left(t, T_{n \vee \eta(t)}\right)$ and $X\left(t, T_{n \vee \eta(t)}\right) \tilde{V}_{n}\left(t, T_{j}\right)$ for all $j \in\{1, \ldots, N-1\}$, all foreign forward LIBORs $\tilde{L}\left(t, T_{j}\right)$ can be recovered. Obviously, one would choose the simulation measure $\mathbf{P}_{T_{n}}$ to match the choice of deterministic forward exchange rate volatility, i.e. such that the link exchange rate $X\left(t, T_{n \vee \eta(t)}\right)$ is lognormal under $\mathbf{P}_{T_{n}}$ up to $T_{n}$, and conditionally lognormal under $\mathbf{P}_{\eta(t)}$ given $\mathcal{F}_{T_{\eta(t)-1}}$ on each interval $\left[T_{\eta(t)-1}, T_{\eta(t)}\right]$ thereafter. Given $X\left(t, T_{n \vee \eta(t)}\right)$, all $L\left(t, T_{j}\right)$ and $\tilde{L}\left(t, T_{j}\right)$, the remaining forward exchange rates can also be recovered.

For the case where all discrete tenor forward exchange rates are assumed to be lognormal under the respective forward measures, a slightly different transformation suggests itself. Set

$$
U_{n}\left(t, T_{j}\right)= \begin{cases}X\left(t, T_{j}\right) \prod_{i=j}^{n-1}\left(1+\delta L\left(t, T_{i}\right)\right) & j=1, \ldots, n-1 \\ X\left(t, T_{j}\right) \prod_{i=n \vee \eta(t)}^{j-1}\left(1+\delta L\left(t, T_{i}\right)\right)^{-1} & j=n, \ldots, N\end{cases}
$$

which is a martingale under the $T_{n}$ forward measure up to $T_{n}$, and under the spot LIBOR measure thereafter. Simulating $U_{n}\left(t, T_{j}\right)$ for all $j \in\{1, \ldots, N\}$ and $V_{n}\left(t, T_{j}\right)$ for all $j \in$ $\{1, \ldots, N-1\}$ yields all $L\left(t, T_{j}\right)$ and all $X\left(t, T_{j}\right)$, from which all $\tilde{L}\left(t, T_{j}\right)$ can then be recovered.

Alternatively to the algorithm by Glasserman/Zhao, one can make use of the relationships between domestic and foreign forward measures derived in section 1 to transform Brownian motion increments generated under the measure chosen for simulation into the corresponding Brownian motion increments for all other measures, following the approach proposed in Brace, Musiela and Schlögl (1998b). Each of the rates for which deterministic volatility is assumed is then simulated as a lognormal martingale under the corresponding forward measure. Again, only these rates need to be simulated, as all others can be recovered by means of static relationships such as (6) and (9), i.e.

$$
X\left(t, T_{i}\right)=X\left(t, T_{i+1}\right) \frac{1+\delta \tilde{L}\left(t, T_{i}\right)}{1+\delta L\left(t, T_{i}\right)}
$$

\footnotetext{
${ }^{21}$ Glasserman and Zhao's algorithm is formulated in terms of the discrete tenor model. However, it is also directly applicable to the continuous tenor version of the model proposed in Schlögl (1999), since in that case all Markovian properties of the discrete tenor model are preserved.
} 


\section{Conclusion}

Applying a Black/Scholes-type formula to price a derivative financial instrument relies on the lognormality of the underlying asset under the appropriate martingale measure. When simultaneously considering interest rate and currency risk, the choice of lognormality assumptions is restricted by no-arbitrage conditions resulting from the relationship between forward exchange rates and domestic and foreign interest rates. Postulating a lognormal Market Model for both the domestic and foreign fixed income markets leaves only the possibility of specifying a lognormal probability law for one forward exchange rate, while if only domestic interest rate dynamics are given, all forward exchange rates could be assumed to be lognormal under the respective forward measures. These two cases may be mixed and combined in a variety of ways into "hybrid" models. Conversely, choosing lognormality assumptions so as to facilitate calibration to at-the-money prices of liquidly traded instruments allows less liquid volatilities to be interpolated or extrapolated in a consistent manner, for example using implied volatilities of short-dated currency options and long-dated interest rate options to obtain prices for currency options of longer maturity.

However, the key point to be made by formulating a consistent, arbitrage-free model is not which variables can (simultaneously) have deterministic volatilities and therefore a lognormal probability law under the corresponding martingale measure. Approximations permit the derivation of Black/Scholes-type formulae in many cases where the underlying rates are not strictly lognormal. Rather, the key point is that the model has to reflect the relationships between the volatilities of the underlying variables and between measures associated with various numeraires. These relationships are determined by no-arbitrage constraints and are valid irrespective of the assumptions on the volatilities. Given a particular set of assumptions, they determine the appropriate approximations when evaluating instruments which cannot be priced exactly in closed form.

Two further extensions of the model suggest themselves. They are simple (though in some cases tedious) applications of the basic approach discussed in this paper. For one, the present results easily scale to the case of arbitrarily many currencies. Secondly, for the domestic and/or foreign fixed income market, the lognormality assumption on forward LIBORs may be replaced by a forward swap rate model of the type developed by Jamshidian (1997).

Also, it is worthwhile to stress that the measure relationships derived in section 1 do not depend on assumptions of deterministic volatility functions. ${ }^{22}$ Thus they are equally valid in any term structure model constructed along the lines of Heath, Jarrow and Morton $(1992)^{23}$ as well as in extensions of the Market Models to more general volatility functions, such as the model of level-dependent LIBOR volatilities proposed by Andersen and Andreasen (1998).

\footnotetext{
${ }^{22}$ That no-arbitrage contraints restrict the choice of volatilities and thereby the relationships between martingale measures associated with various numeraires has been previously noted by Andreasen (1995), who relates the spot exchange rate volatility to the risk premia for the component currencies and constructs a consistent multicurrency, multidimensional extension of the Cox, Ingersoll and Ross (1985) model with time dependent parameters.

${ }^{23}$ Other consistent multicurrency models in the literature implicitly satisfy the restrictions derived here. This includes the model of Andreasen (1995), as well as Frey and Sommer (1996). However, in the latter case all volatility functions in equation (11) are deterministic, i.e. lognormal exchange rate dynamics and lognormal bond price dynamics in all currencies can consistently coexist in a multicurrency Gaussian HJM model.
} 
In summary, while this paper makes no attempt to develop a model to better explain the empirical dynamics of interest rates and exchange rates, we have identified the trade-offs between lognormality assumptions supporting the simultaneous use of Black/Scholes-type formulae for various derivative financial instruments, showing to what degree a model can reflect popular market practice while remaining consistent with no-arbitrage.

\section{APPEndix A}

Proof of lemma 3.3: By the definition of the two measures, we must have

$$
\left.\frac{d \mathbf{P}_{\rho}}{d \mathbf{P}_{\beta}}\right|_{\mathcal{F}_{t}}=\frac{\rho(t)}{\rho(0)} \frac{\beta(0)}{\beta(t)}=\frac{\rho(t)}{\beta(t)} \quad \mathbf{P}_{\beta} \text {-a.s. }
$$

If the volatility of $B\left(t, T_{\eta(t)}\right)$ is zero for all $T_{0} \leq t \leq T_{N}$, we have ${ }^{24}$

$$
B\left(t, T_{\eta(t)}\right)=\frac{\beta(t)}{\beta\left(T_{\eta(t)}\right)}=\exp \left\{\int_{t}^{T_{\eta(t)}} r(s) d s\right\} \quad \forall T_{0} \leq t \leq T_{N}
$$

and consequently

$$
\begin{aligned}
\frac{\rho(t)}{\beta(t)} & =\beta(t)^{-1} B\left(t, T_{\eta(t)}\right) \prod_{i=0}^{\eta(t)-1} B\left(T_{i}, T_{i}+\delta\right)^{-1} \\
& =\beta(t)^{-1} \frac{\beta(t)}{\beta\left(T_{\eta(t)}\right)} \prod_{i=0}^{\eta(t)-1} \frac{\beta\left(T_{i+1}\right)}{\beta\left(T_{i}\right)} \\
& =\beta\left(T_{0}\right)^{-1}=1
\end{aligned}
$$

Thus

$$
\left.\frac{d \mathbf{P}_{\rho}}{d \mathbf{P}_{\beta}}\right|_{\mathcal{F}_{t}}=1 \quad \forall T_{0} \leq t \leq T_{N}, \mathbf{P}_{\beta}-\text { a.s. }
$$

and the two measures coincide.

The following lemma shows that the spot LIBOR measure may be interpreted as the measure obtained by "chaining" conditional probabilities of forward measures:

A.1. Lemma. For all $\mathcal{F}_{T_{i+1}}$-measurable events, the conditional probabilities given $\mathcal{F}_{T_{i}}$ are identical for the spot LIBOR measure and the time $T_{i+1}$ forward measure, i.e.

$$
\mathbf{P}_{\rho}\left\{A \mid \mathcal{F}_{T_{i}}\right\}=\mathbf{P}_{T_{i+1}}\left\{A \mid \mathcal{F}_{T_{i}}\right\} \quad \forall i \in\{0, \ldots, N-1\}, A \in \mathcal{F}_{T_{i+1}}
$$

\footnotetext{
${ }^{24}$ cf. remark 2.1 of Brace, Gatarek and Musiela (1997)
} 
Proof. Let $E_{\rho}$ and $E_{T_{i+1}}$ denote the expectation operators under the measures $\mathbf{P}_{\rho}$ and $\mathbf{P}_{T_{i+1}}$, respectively.

$$
\begin{aligned}
\mathbf{P}_{\rho}\left\{A \mid \mathcal{F}_{T_{i}}\right\} & =E_{\rho}\left[I_{A} \mid \mathcal{F}_{T_{i}}\right] \\
= & \frac{E_{T_{i+1}}\left[I_{A} \cdot\left(\left.\frac{d \mathbf{P}_{\rho}}{d \mathbf{P}_{T_{i+1}}}\right|_{\mathcal{F}_{T_{i+1}}}\right) \mid \mathcal{F}_{T_{i}}\right]}{E_{T_{i+1}}\left[\left(\left.\frac{d \mathbf{P}_{\rho}}{d \mathbf{P}_{T_{i+1}}}\right|_{\mathcal{F}_{T_{i+1}}}\right) \mid \mathcal{F}_{T_{i}}\right]} \\
= & \frac{B\left(0, T_{i+1}\right)\left(\prod_{j=0}^{i}\left(1+\delta L\left(T_{j}, T_{j}\right)\right)\right) E_{T_{i+1}}\left[I_{A} \mid \mathcal{F}_{T_{i}}\right]}{B\left(0, T_{i+1}\right)\left(\prod_{j=0}^{i}\left(1+\delta L\left(T_{j}, T_{j}\right)\right)\right)} \\
= & E_{T_{i+1}}\left[I_{A} \mid \mathcal{F}_{T_{i}}\right]=\mathbf{P}_{T_{i+1}}\left\{A \mid \mathcal{F}_{T_{i}}\right\}
\end{aligned}
$$

\section{REFERENCES}

Andersen, L. and Andreasen, J. (1998), Volatility Skews and Extensions of the LIBOR Market Model, General Re Financial Products, working paper .

Andreasen, J. F. (1995), Pricing by Arbitrage in an International Economy, Research in International Business and Finance 12, 93-116.

Black, F. and Scholes, M. (1973), The Pricing of Options and Corporate Liabilities, Journal of Political Economy pp. 637-654.

Brace, A. and Womersley, R. S. (2000), Exact Fit to the Swaption Volatility Matrix Using Semidefinite Programming, ICBI Global Derivatives Conference, working paper.

Brace, A., Dun, T. A. and Barton, G. (1998a), Towards a Central Interest Rate Model, Conference Global Derivatives '98, working paper.

Brace, A., Gatarek, D. and Musiela, M. (1997), The Market Model of Interest Rate Dynamics, Mathematical Finance 7(2), 127-155.

Brace, A., Musiela, M. and Schlögl, E. (1998b), A Simulation Algorithm for Lognormal Market Models, The University of New South Wales, working paper .

Cox, J. C., Ingersoll, J. E. and Ross, S. A. (1985), An Intertemporal General Equilibrium Model of Asset Prices, Econometrica 53(2), 363-384.

Dun, T. A., Schlögl, E. and Barton, G. (2001), Simulated Swaption Delta-Hedging in the Lognormal Forward LIBOR Model, International Journal of Theoretical and Applied Finance p. (forthcoming).

Frey, R. and Sommer, D. (1996), A Systematic Approach to Pricing and Hedging International Derivatives with Interest Rate Risk: Analysis of International Derivatives Under Stochastic Interest Rates, Applied Mathematical Finance 3(4), 295-317.

Geman, H. (1989), The Importance of the Forward Neutral Probability in a Stochastic Approach to Interest Rates, ESSEC, working paper.

Geman, H., El Karoui, N. and Rochet, J.-C. (1995), Changes of Numeraire, Changes of Probability Measure and Option Pricing, Journal of Applied Probability 32, 443-458.

Glasserman, P. and Zhao, X. (2000), Arbitrage-Free Discretization of Lognormal Forward Libor and Swap Rate Models, Finance and Stochastics.

Harrison, J. M. and Kreps, D. M. (1979), Martingales and Arbitrage in Multiperiod Securities Markets, Journal of Economic Theory 20,381-408.

Harrison, J. M. and Pliska, S. R. (1981), Martingales and Stochastic Integrals in the Theory of Continuous Trading, Stochastic Processes and their Applications 11, 215-260.

Harrison, J. M. and Pliska, S. R. (1983), A Stochastic Calculus Model of Continuous Trading: Complete Markets, Stochastic Processes and their Applications 15, 313-316.

Heath, D., Jarrow, R. and Morton, A. (1992), Bond Pricing and the Term Structure of Interest Rates: A New Methodology for Contingent Claims Valuation, Econometrica 60(1), 77-105. 
Jamshidian, F. (1987), Pricing of Contingent Claims in the One-Factor Term Structure Model, Financial Strategies Group, Merrill Lynch Capital Markets, working paper.

Jamshidian, F. (1997), LIBOR and Swap Market Models and Measures, Finance and Stochastics $\mathbf{1}(4), 293-330$.

Mikkelsen, P. (1999), Pricing Interest Rate and Foreign Exchange Derivatives in a Consistent LIBOR Market Model, Aarhus School of Business, working paper.

Miltersen, K. R., Sandmann, K. and Sondermann, D. (1997), Closed Form Solutions for Term Structure Derivatives with Log-Normal Interest Rates, The Journal of Finance 52(1), 409-430.

Musiela, M. and Rutkowski, M. (1997a), Continuous-Time Term Structure Models: A Forward Measure Approach, Finance and Stochastics.

Musiela, M. and Rutkowski, M. (1997b), Martingale Methods in Financial Modelling, Vol. 36 of Applications of Mathematics, Springer-Verlag, New York, New York, USA.

Pedersen, M. B. and Miltersen, K. R. (2000), Dual-Currency LIBOR Market Models, Financial Research Department, SimCorp A/S, working paper draft.

Rady, S. (1997), Option Pricing in the Presence of Natural Boundaries and a Quadratic Diffusion Term, Finance and Stochastics 1(4), 331-344.

Sandmann, K. and Sondermann, D. (1994), A Term Structure Model and the Pricing of Interest Rate Options, The Review of Futures Markets 12(2), 391-423.

Sandmann, K., Sondermann, D. and Miltersen, K. (1995), Closed Form Term Structure Derivatives in a Heath-Jarrow-Morton Model with Log-normal Annually Compounded Interest Rates, Research Symposium Proceedings CBOT pp. 145-164.

Schlögl, E. (1999), Some Remarks on Arbitrage Free Term Structure Interpolation in the Lognormal Interest Rate Market Models, University of Technology, Sydney, working paper. 